\title{
Análise sobre os conceitos e práticas de Gestão por Processos: revisão sistemática e bibliometria
}

\section{Analysis of business process management theory and practices: systematic literature review and bibliometrics}

\author{
Diego Rodrigues Iritani ${ }^{1}$ \\ Sandra Naomi Morioka ${ }^{2}$ \\ Marly Monteiro de Carvalho \\ Aldo Roberto Ometto
}

\begin{abstract}
Resumo: Este artigo tem como objetivo analisar a teoria sobre Gestão por Processos de Negócio (BPM - Business Process Management). Para isso foi realizada uma revisão bibliográfica sistemática e foram utilizadas técnicas de análise de publicações, citações e de bibliometria. Os resultados mostram a multidisciplinaridade de BPM, como as diferentes áreas de pesquisa compreendem o tema e como praticam essa abordagem. Dentre os periódicos relevantes para o tema, destacaram-se o Business Process Managament Journal, Knowlegde and Process Management e Total Quality Management \& Business Excellence. Dentre as abordagens de aplicação foi possível verificar que existem três tipos adotados pelo estudo e ao todo foram identificadas oito práticas de BPM. As redes de bibliometria mostraram a existência de agrupamentos bibliométricos que pouco interagem, e a relação de BPM com as abordagens de gestão da qualidade e reengenharia. Apesar do aumento de publicações na área, o que mostra a relevância do tema, as publicações tendem a seguir escolas distintas e que ainda atuam de forma isolada na área de BPM.
\end{abstract}

Palavras-chave: Gestão por processos de negócio. Gestão organizacional. Análise bibliométrica. Práticas de BPM.

\begin{abstract}
This article aims to analyze the theory on the subject of Business Process Management (BPM). To this end, a systematic literature review, whose purpose was to analyze BPM practices, was conducted through the analysis of publications, citations and bibliometrics. The results show the multidisciplinarity of BPM, and how different areas of research address the theme and practice this approach. Among the relevant journals on the topic, the following are highlighted: Business Process Management Journal, Knowlegde and Process Management and Total Quality Management \& Business Excellence. Regarding the application approaches, we found that there are three types adopted by the study, and eight practices of BPM were identified. Networks of bibliometrics showed the existence of bibliometric groups that interact very weakly, and the relationship of BPM with the approaches of quality management and reengineering. The increasing number of publications in the area and the existence of several areas of research interested in the topic justify the analysis of the concepts and practices of BPM.
\end{abstract}

Keywords: Business process management. Organizational management. Bibliometric analysis. BPM practices.

\section{Introdução}

A gestão por processos de negócio (Business Process Management-BPM) pode ser compreendida como uma abordagem para identificar, desenhar, executar, documentar, medir, monitorar, controlar e melhorar os processos de negócio para que os resultados desejados possam ser alcançados (ASSOCIATION..., 2009). Os benefícios de se adotar essa abordagem incluem: maior velocidade nas melhorias e mudanças de mercado, aumento da satisfação do consumidor, melhor qualidade de produtos, redução de custos e maior compreensão sobre as atividades da organização (KOHLBACHER, 2010). Entretanto, a aplicação de BPM passa pelo entendimento e apreciação de suas práticas, que normalmente são estruturadas em um ciclo de vida (KO et al., 2009).

Segundo Harmon (2010), BPM tem origem em três abordagens: a de gestão de negócio; a de gestão da qualidade total e a de tecnologia da informação. Embora existam trabalhos holísticos como o de Harmon (2010), ainda existem divergências na literatura sobre os conceitos e práticas de BPM,

${ }^{1}$ Departamento de Engenharia de Produção, Escola de Engenharia de São Carlos - EESC, Universidade de São Paulo - USP, CEP 13566-590, São Carlos, SP, Brasil, e-mail: diritani@yahoo.com.br; aometo@sc.usp.br

${ }^{2}$ Departamento de Engenharia de Produção, Escola Politécnica - POLI, Universidade de São Paulo - USP, CEP 05508-010,

São Paulo, SP, Brasil, e-mail: sanmorioka@gmail.com; marlymc@usp.br 
principalmente devido à sua origem multidisciplinar (DEBRUIN \& DOEBELI, 2009). Nesse sentido, o presente estudo visa contribuir com um maior entendimento sobre as origens e tendências da área de BPM, bem como vislumbrar oportunidades de contribuição entre essas diferentes perspectivas de BPM. Assim, este artigo tem como objetivo apresentar e discutir o panorama da produção científica sobre BPM.

A primeira seção apresenta a contextualização e o objetivo desta pesquisa. A segunda seção apresenta os métodos de pesquisa, que compreenderam a revisão bibliográfica sistemática e os métodos de análise de publicações, citações e bibliometria. $\mathrm{Na}$ seção seguinte são apresentados os resultados de pesquisa, que incluem a evolução das publicações, os periódicos de maior destaque, as áreas representativas dentro do tema, trabalhos mais citados, os agrupamentos bibliométricos e as referências de destaque. Por fim, a última seção apresenta as conclusões do estudo, suas limitações e sugestões para trabalhos futuros.

\section{Metodologia de pesquisa}

Segundo Biolchinni et al. (2005), a revisão bibliográfica sistemática (RBS) é uma abordagem de pesquisa que possui etapas bem definidas, planejadas de acordo com protocolo e objetivos previamente estabelecidos. Dentre as características dessa metodologia cabe destacar: definição de estratégia de revisão na fase de planejamento; avaliação crítica dos estudos; documentação das atividades e resultados; capacidade de síntese dos estudos selecionados; busca abrangente e exaustiva sobre determinado assunto; critérios de qualificação reproduzíveis e claros para seleção dos estudos.

O processo de revisão bibliográfica sistemática proposto por Biolchinni et al. (2005) possui três etapas: planejamento, execução e análise dos resultados. Entre as etapas são previstas avaliações que visam aprovar ou reprovar as atividades e resultados obtidos. A etapa de planejamento é composta por duas atividades: a identificação da necessidade de uma revisão e a criação do protocolo de revisão. É durante a identificação da necessidade de revisão que os objetivos da revisão são definidos. O protocolo, por sua vez, é composto pelos objetivos da revisão e pelos métodos e procedimentos a serem utilizados na revisão sistemática, dentre os quais se destacam: a seleção das bases de dados, os critérios de inclusão dos estudos, os critérios de qualificação das bases de dados, a definição do idioma e dos tipos de estudo, os procedimentos para busca e seleção dos estudos, os procedimentos para extração dos dados e a definição dos métodos para análise dos resultados.

\subsection{Execução da revisão bibliográfica sistemática}

As bases de dados utilizadas nesta pesquisa foram a ISI Web of Knowledge (Web of Science) e SciVerse Scopus, pois são bases indexadas e que permitem a exportação de metadados necessários para as análises de publicações, citações e bibliometria. A string de busca utilizada foi "Business Process Management", resultando em uma amostra de 3.521 publicações de áreas temáticas e de natureza relacionadas principalmente a Ciência da Computação, Administração, Engenharia e Gestão da Qualidade.

Em seguida foram aplicados dois filtros de seleção. $\mathrm{O}$ primeiro foi o relacionado ao tipo de documento, no qual foram selecionados somente artigos, uma vez que somente esses passam por processos de avaliação por pares na sua versão completa. Após a aplicação desse filtro, a amostra foi reduzida a 1.033 artigos, que foram submetidos ao filtro de seleção, que compreendeu a aplicação do critério de inclusão por meio da leitura do título, do resumo e das palavras-chave. O critério de inclusão adotado nesse estudo compreendeu abordar a temática de BPM. A amostra de artigos foi reduzida a 879, dos quais 233 artigos estavam duplicados nas bases de dados, resultando em uma amostra final de 646 artigos. Essa amostra foi submetida a análise de publicações, citações e bibliometria, na qual essas metodologias direcionaram a discussão dos artigos por classificação, número de citações e indicadores bibliométricos.

Após a definição da amostra, foram extraídos os metadados disponíveis nas bases de dados ISI Web of Science e Scopus como: resumo, autores, palavras-chave, periódico, referências, número de citações, entre outros dados. Os metadados foram então importados para o software Sci²Tool, versão 0.5 alpha (SCI2..., 2009), no qual foram elaboradas e analisadas as redes de bibliometria.

\subsection{Análise de publicações}

A primeira análise de publicações foi a de periódicos por ano, a qual permitiu identificar os periódicos com maior número de publicações, bem como a tendência das publicações ao longo dos anos.

Em seguida, as publicações foram classificadas quanto à abordagem de aplicação e às práticas de BPM. A classificação quanto à abordagem de aplicação foi realizada com base no trabalho de Debruin \& Doebeli (2009), que mencionam três tipos de abordagens para BPM: abordagem com foco em tecnologias e sistemas de informação; abordagem com foco na gestão e melhoria dos processos de negócio; e abordagem com foco na gestão organizacional por processo de negócio 
(Quadro 1). A classificação quanto às práticas de BPM foi realizada por meio de um levantamento preliminar na literatura, no qual foram identificadas oito práticas de BPM: planejamento de BPM; modelagem de processos; análise de processos; melhoria e mudanças de processos; medição, monitoramento e controle de processos; simulação de processos; implementação de processos/sistemas de apoio; e modelos de ciclo de vida de BPM. O Quadro 2 mostra a descrição das oito práticas de BPM adotadas neste estudo.

\subsection{Análise de citações}

A análise de citações apresentou três atividades: cálculo do índice corrigido de citações dos artigos, ordenação dos artigos mais citados e análise gráfica dos 20 artigos mais citados. A Equação 1 mostra o cálculo do índice corrigido (ICc), onde IC é o índice de citações extraído da base de dados e FI é o fator de impacto do periódico em que o artigo foi publicado. A correção do índice de citação tem como objetivo incluir, além do número de citações do artigo, a

Quadro 1. Abordagens de aplicação de BPM.

\begin{tabular}{|l|l|}
\hline Abordagem de aplicação de BPM & \multicolumn{1}{c|}{ Descrição } \\
\hline $\begin{array}{l}\text { Tecnologia e sistemas de } \\
\text { informação }\end{array}$ & $\begin{array}{l}\text { Entende BPM como uma solução para negócios utilizando sistemas de } \\
\text { software ou tecnologias para automatizar e gerenciar os processos de negócio. }\end{array}$ \\
\hline $\begin{array}{l}\text { Gestão e melhoria de processos de } \\
\text { negócio }\end{array}$ & $\begin{array}{l}\text { Entende BPM como uma abordagem para gerenciar e melhorar os processos } \\
\text { de negócio com foco em seu ciclo de vida. }\end{array}$ \\
\hline $\begin{array}{l}\text { Gestão da organização por } \\
\text { processos de negócio }\end{array}$ & $\begin{array}{l}\text { Entende BPM como uma abordagem para gerenciar uma organização por } \\
\text { meio da visão de processos de negócio (foco vai além dos processos de } \\
\text { negócio). }\end{array}$ \\
\hline
\end{tabular}

Quadro 2. Abordagens de aplicação de BPM.

\begin{tabular}{|c|c|}
\hline Prática de BPM & Descrição \\
\hline Planejamento de BPM & $\begin{array}{l}\text { Tem o propósito de definir as atividades de gestão por processos que contribuirão para o } \\
\text { alcance das metas da organização por meio de análises dos ambientes internos e externos, } \\
\text { estabelecimento de estratégias e abordagens para mudança, definição de planos de ação } \\
\text { para implantação de BPM e priorização de processos de negócio (ASSOCIATION..., } \\
\text { 2009; VALLE \& OLIVEIRA, 2009). }\end{array}$ \\
\hline $\begin{array}{l}\text { Modelagem de } \\
\text { processos }\end{array}$ & $\begin{array}{l}\text { Tem como objetivo documentar e registrar os processos de negócio em modelos. Além } \\
\text { disso, os processos podem ser modelados para prover dados de integração entre processos, } \\
\text { para fins de Tecnologia da Informação (TI), da Qualidade e outros (ASSOCIATION..., } \\
\text { 2009; VALLE \& OLIVEIRA, 2009). }\end{array}$ \\
\hline Análise de processos & $\begin{array}{l}\text { Incorpora metodologias com a finalidade de entender os processos atuais no contexto das } \\
\text { metas e objetivos estabelecidos pela organização, assimilando informações oriundas de } \\
\text { planos estratégicos, modelos de processos e medição de desempenho a fim de entender os } \\
\text { processos de negócio no escopo da organização como um todo (ASSOCIATION..., 2009; } \\
\text { VALLE \& OLIVEIRA, 2009). }\end{array}$ \\
\hline $\begin{array}{l}\text { Melhoria e mudança de } \\
\text { processos }\end{array}$ & $\begin{array}{l}\text { Compreende práticas como uso de metodologias para otimizar, inovar e redesenhar os } \\
\text { processos de negócio (SMART et al., 2009; VALLE \& OLIVEIRA, 2009). }\end{array}$ \\
\hline $\begin{array}{l}\text { Medição, } \\
\text { monitoramento e } \\
\text { controle de processos }\end{array}$ & $\begin{array}{l}\text { Atividades que visam mensurar e monitorar os processos de negócio como, por exemplo: } \\
\text { registrar e controlar o desempenho dos processos, retroalimentar o Planejamento e } \\
\text { Modelagem de Processos (ASSOCIATION..., 2009; VALLE \& OLIVEIRA, 2009). }\end{array}$ \\
\hline $\begin{array}{l}\text { Simulação de } \\
\text { processos }\end{array}$ & $\begin{array}{l}\text { Prática diretamente relacionada à modelagem e análise de processos cujo objetivo é } \\
\text { simular, por meio da execução de modelos de processos, diversos cenários (HAN et al., } \\
\text { 2009). }\end{array}$ \\
\hline $\begin{array}{l}\text { Implementação de } \\
\text { processos e sistemas } \\
\text { de apoio }\end{array}$ & $\begin{array}{l}\text { Engloba as atividades que garantem o suporte à implementação e à execução dos } \\
\text { processos como. por exemplo, o suporte à implementação de novos processos e } \\
\text { de testes de soluções, implementação de planos de transferência de tecnologias } \\
\text { (ASSOCIATION..., 2009; VALLE \& OLIVEIRA, 2009). }\end{array}$ \\
\hline $\begin{array}{l}\text { Modelos de ciclo de } \\
\text { vida de BPM }\end{array}$ & $\begin{array}{l}\text { Consiste na prática de adotar modelos de referência iterativos para a gestão dos processos } \\
\text { de negócio. As atividades desses modelos normalmente são: planejamento, modelagem, } \\
\text { análise, melhoria e medição de processos de negócio. Outra característica são a melhoria } \\
\text { contínua e a retroalimentação do plano estratégico da organização (ASSOCIATION..., } \\
\text { 2009; VALLE \& OLIVEIRA, 2009). }\end{array}$ \\
\hline
\end{tabular}


relevância do periódico em que o artigo foi publicado (LOPES \& CARVALHO, 2012):

$$
I C c=I C \times(F I+1)
$$

Em seguida foram executadas as atividades relativas à análise das redes de bibliometria, que incluem a importação de dados para o software $\mathrm{Sci}^{2}$ Tool, extração das redes de bibliometria, pré-processamento e tratamento das redes, análise das redes e visualização, edição e elaboração das redes. Segundo Garfield (2001), a análise bibliométrica permite identificar similaridades e grupos de interesse, bem como mostrar visões prospectivas e retrospectivas em uma determinada área de pesquisa. Além disso, por meio da análise bibliométrica é possível analisar o fluxo de informação e a relação entre grupos, identificar padrões e tendências em uma área e as pesquisas mais centrais e sua contribuição para o corpo de conhecimento científico (BORNER et al., 2007).

A extração das redes de bibliometria compreendeu a extração das redes de artigos para referência e cocitação. A Figura 1 ilustra os mecanismos e o conceito das redes utilizadas nesta pesquisa (GIPP \& BEEL, 2009; GARFIELD, 2001). A rede de artigos para referência conecta os artigos da amostra com as referências mais citadas e tem como propósito identificar possíveis referências importantes. A segunda rede elaborada foi a de cocitação. A rede de cocitação indica o grau de similaridade entre as referências, pois mostra os pares de referências citados por um artigo da amostra. A análise das redes de cocitação auxilia na compreensão da estrutura intelectual da área e permite identificar afinidades temáticas dos pesquisadores, bem como traça um panorama prospectivo do tema estudado (CULNAN et al., 1990; PILKINGTON \& LISTON-HEYES, 1999; CARVALHO et al., 2013; GARFIELD, 2001). Para essa rede foram calculados três indicadores

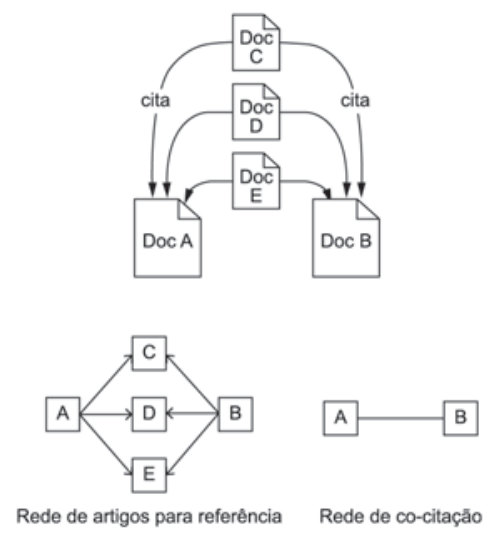

Figura 1. Mecanismo de citação e redes de bibliometria utilizados nesta pesquisa. Fonte: Adaptado de Garfield (2001) e Gipp \& Beel (2009). bibliométricos: densidade da rede, centralidade e índice de intermediação.

- Densidade da rede: razão entre as relações existentes e o total de relações possíveis de ocorrer;

- Centralidade: é a relação adjacente de um ator com os demais atores da rede;

- Índice de intermediação: possibilidade que um autor tem para intermediar as comunicações entre pares de atores, que não se relacionam diretamente.

\section{Resultados da pesquisa}

As seções seguintes apresentam os principais resultados da revisão bibliográfica sistemática, das análises de publicações, citações e bibliometria dos artigos que abordam a temática de BPM.

\subsection{Análise de publicações}

A Figura 2 apresenta o gráfico de publicações por ano em função das bases de dados em que as publicações foram encontradas.

A Tabela 1 mostra a relação de publicações por periódico por ano. Dentre os periódicos com maior número de publicações, os que estão relacionados com a abordagem de gestão são:

- Business Process Management Journal: Aborda BPM como tema central. O escopo desse periódico inclui publicações com foco na gestão do conhecimento, métodos de modelagem e análise de processos de negócio, melhores práticas em BPM, gestão de desempenho, ferramentas de gestão de mudança, dentre outros.

- Knowlegde and Process Management: Publicações na área de gestão do conhecimento, aprendizagem organizacional e gestão de processos. Além disso, esse periódico aborda a gestão de desempenho e melhoria de negócio por meio de novas abordagens de gestão, com destaque para publicações do tipo estudo de caso e surveys.

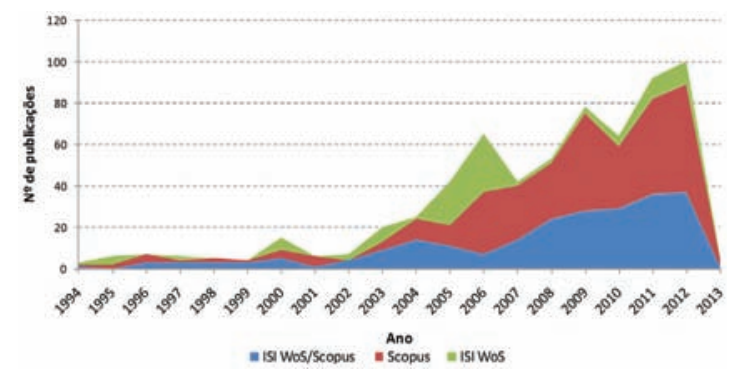

Figura 2. Publicações por ano em função das bases de dados. 


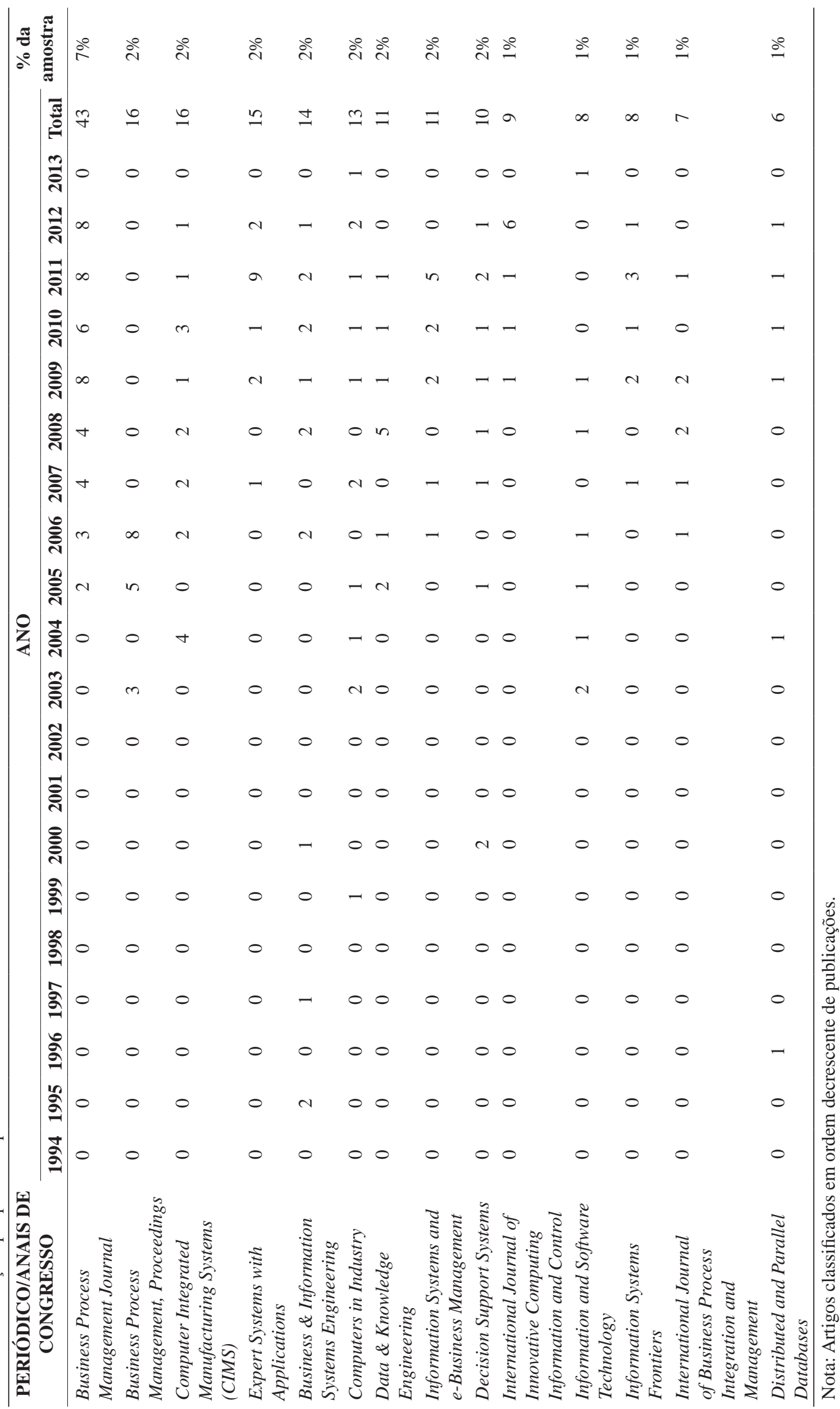




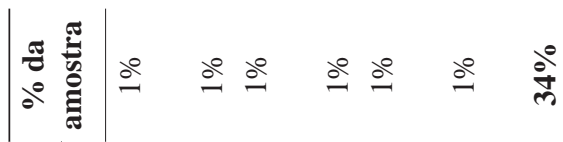

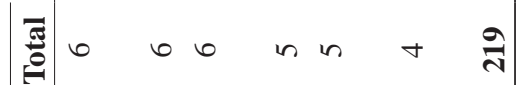

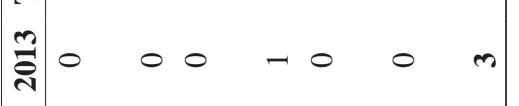

$$
\begin{aligned}
& \text { ปิ) } \\
& \text { ב⿳亠口冋. }
\end{aligned}
$$

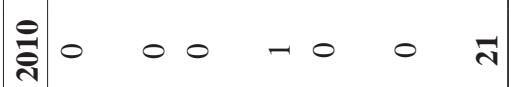

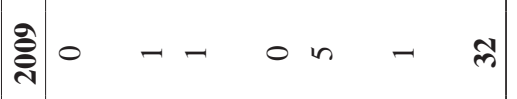

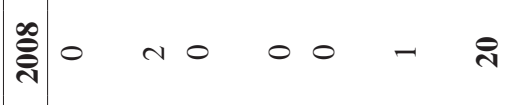

$$
\begin{aligned}
& \text { 今్ }
\end{aligned}
$$

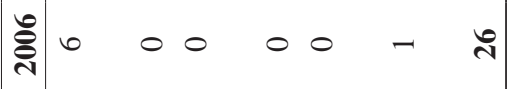

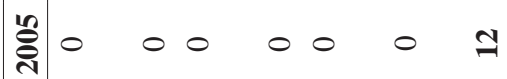

$$
\begin{aligned}
& \text { 岁 } \\
& \text { 气ิి } 00000001 \\
& \text { 气ิેి } 0000000 \\
& \text { हิ) } 0000000
\end{aligned}
$$

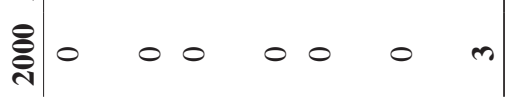

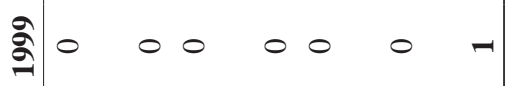

$$
\begin{aligned}
& \text { की० } 0000000
\end{aligned}
$$

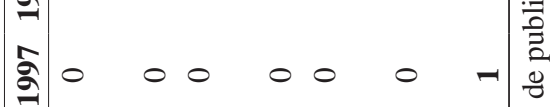

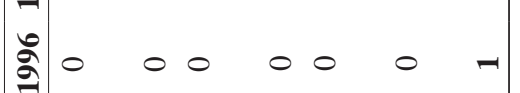

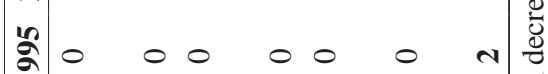

$$
\begin{aligned}
& \begin{array}{llllll}
2 & 0 & 0 & 0 & 0 & 0
\end{array} \\
& \text { హิ }
\end{aligned}
$$

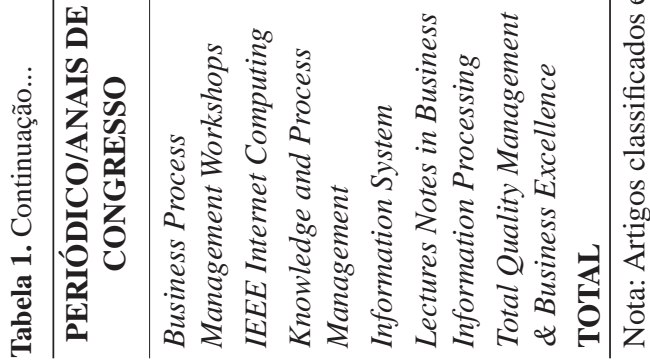


- Total Quality Management \& Business Excellence: Esse periódico aborda como tema central a abordagem Total Quality Management (TQM) que, segundo Garvin (1995), é uma das origens de BPM. De acordo com o autor, ambas as abordagens têm como princípio o foco na satisfação do cliente e a melhoria contínua

A Figura 3 mostra os resultados da classificação das publicações quanto à abordagem de aplicação de BPM. Foi possível notar que a maior parte dos artigos adota a abordagem de BPM como uma abordagem de tecnologia e sistemas de informação (código A1), na qual BPM é vista como uma solução para negócio utilizando sistemas de software ou tecnologias para automatizar e gerenciar os processos de negócio (DEBRUIN \& DOEBELI, 2009). As abordagens de BPM para gerenciar o ciclo de vida de processos de negócio (código A2) e para gerenciar a organização como um todo (código A3) apresentaram menor volume de publicações mas, a partir de 2006, essas publicações apresentaram um aumento considerável no número total de publicações (Figura 3b).

A Figura 4a mostra a evolução das publicações classificadas em função das práticas de BPM. Ao todo foram identificadas oito práticas de
BPM. Os resultados mostram um crescimento acentuado de aplicações de práticas BPM a partir de 2003, acompanhando a tendência do aumento de publicações. A Figura $4 \mathrm{~b}$ retrata a representatividade de cada prática de BPM, nela se destacam as práticas P2 e P7, que a partir de 2000 representaram no mínimo $50 \%$ da aplicação de práticas de BPM. É possível associar esse resultado ao foco das publicações da área de Ciência de Computação, as quais fazem uso de métodos de modelagem de processos para a implementação de sistemas de BPM. Outra prática que está diretamente relacionada a essa área de pesquisa é a P6. A Figura 4b mostra que estudos sobre essa prática começaram a ser publicados em 2003, pois essa é uma atividade que depende diretamente da aplicação computacional e do desenvolvimento de tecnologias de hardware.

O planejamento de BPM (P1), segundo Valle \& Oliveira (2009), tem como objetivo definir as atividades de BPM que contribuirão para o alcance das metas organizacionais. Para isso, o planejamento de BPM inclui: compreensão do ambiente externo e interno e da estratégia organizacional; estabelecimento da estratégia de BPM com foco nas mudanças dos processos; seleção

(a)
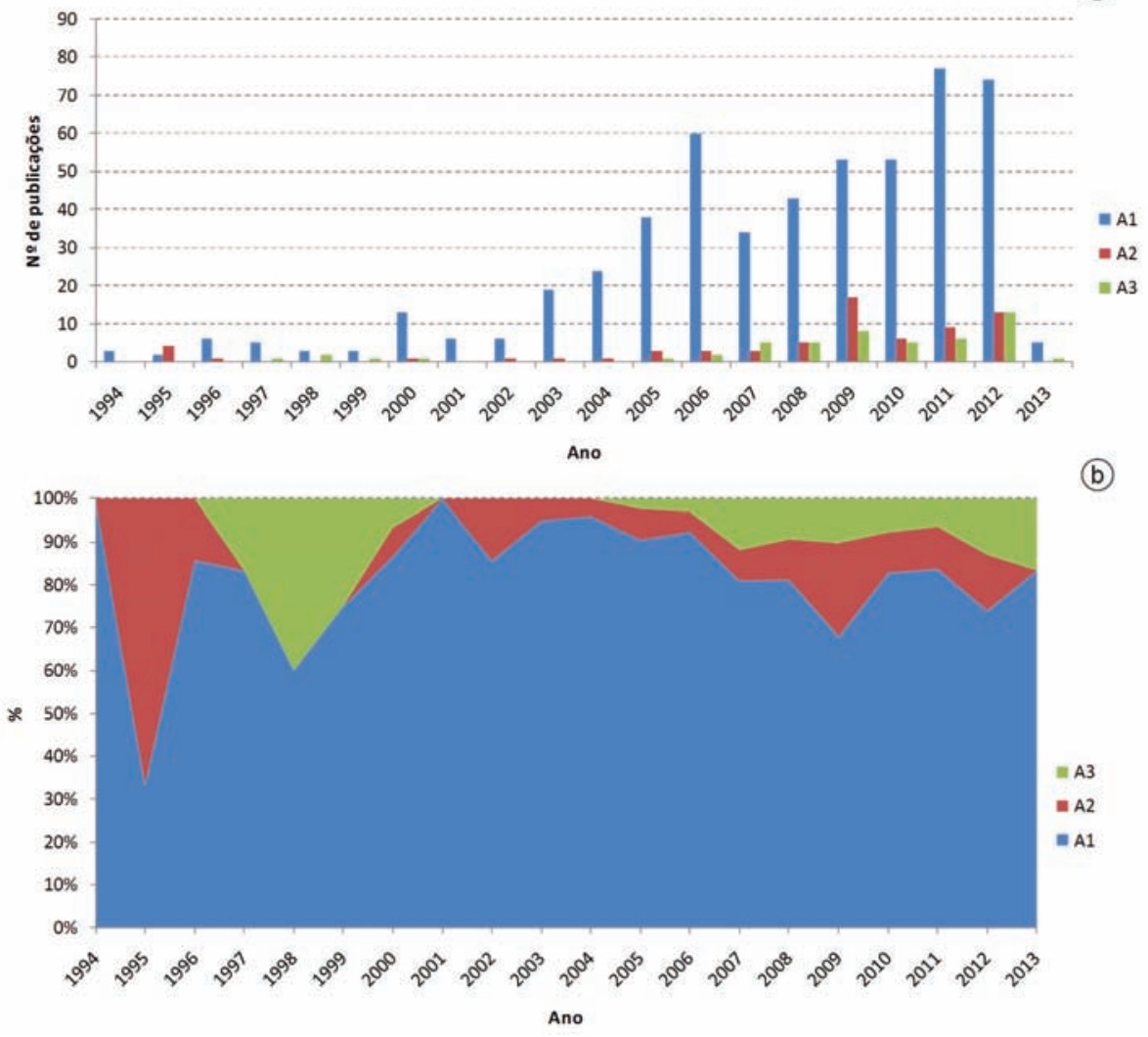

Figura 3. Publicações por categoria de abordagem de aplicação de BPM por ano, em quantidade de publicações (a) e percentual (b). 
(a)
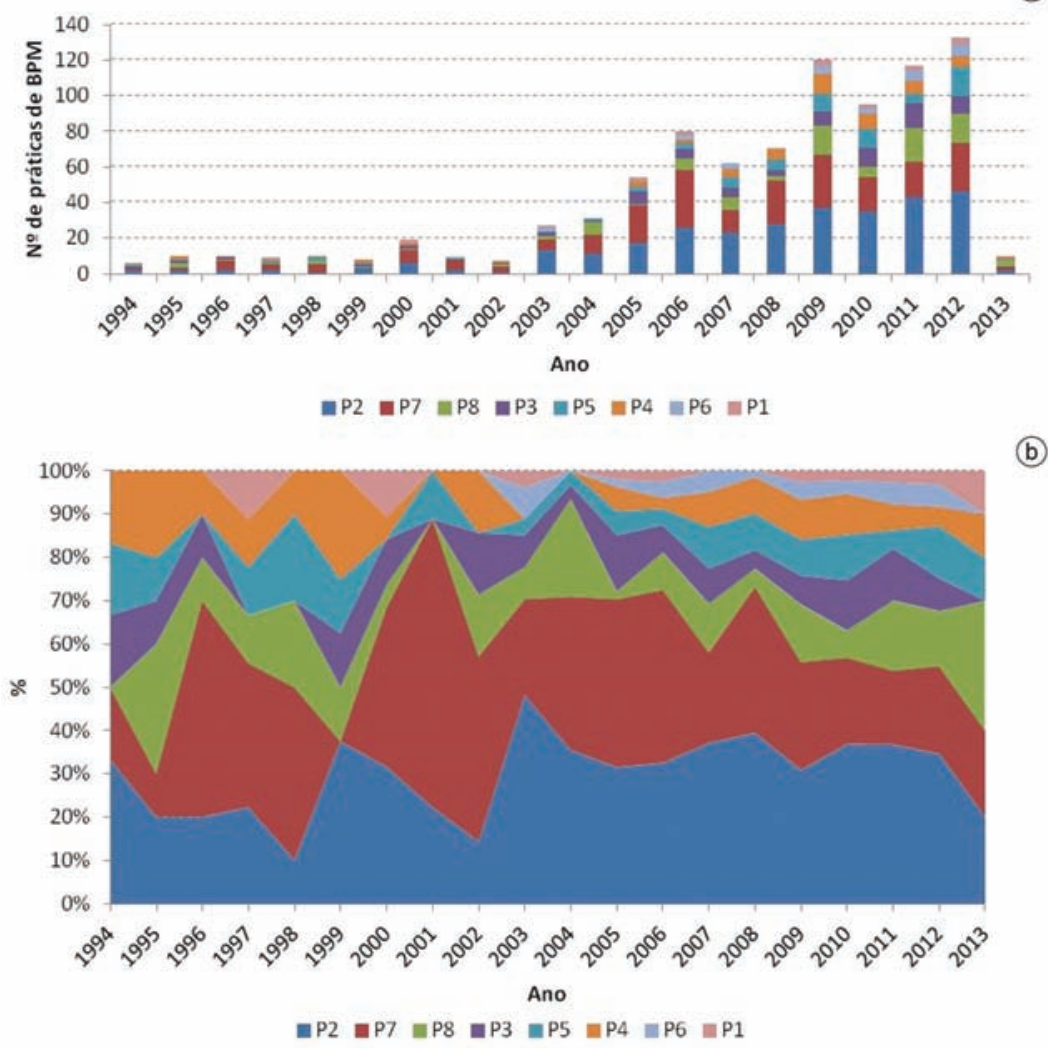

Figura 4. Práticas de BPM abordadas nas publicações por ano, em quantidade (a) e percentual (b). Nota: P1 = planejamento de BPM; P2 = modelagem de processos; P3 = análise de processos; P4 = melhoria e mudança de processos; P5 = medição, monitoramento e controle de processo; P6 = simulação de processos; P7 = implementação de processos e de sistemas de apoio; P8 = modelos de ciclo de vida.

de ferramentas e técnicas de melhoria; definição do plano de implementação; seleção de processos-chave para a estratégia organizacional; e formação de equipes, planejamento e gerenciamento de projetos de melhoria. Essa prática pode ser entendida como o desdobramento do planejamento estratégico (BOARDMAN, 1997) e, para isso ocorrer, cada processo deve possuir metas relacionadas aos objetivos do planejamento estratégico (SKRINJAR \& TRKMAN, 2013).

A prática de modelagem de processos (P2) está relacionada à documentação e compreensão de como as atividades ocorrem (ASSOCIATION..., 2009). Segundo Davenport (2005), modelar processos envolve a representação das atividades em gráficos que normalmente incluem eventos, estágios e propriedades. A modelagem de processos é uma atividade essencial para as demais práticas de BPM, pois todas as atividades seguintes dependem do modelo de processos, que é o principal resultado dessa prática (VOLKNER \& WERNERS, 2000).

A análise de processos (P3) tem como objetivo verificar, validar e identificar potenciais melhorias em processos e incorpora metodologias com a finalidade de entender os processos atuais, com foco nas metas e objetivos estabelecidos pelo planejamento de BPM (PEDRINACI et al., 2008). Para isso, são utilizados informações do planejamento de BPM, dos modelos de processos, da medição de desempenho e da análise de fatores internos e externos (ASSOCIATION..., 2009).

A prática de melhoria de processos $(\mathrm{P} 4)$ relaciona-se principalmente com a prática de modelagem, análise e implementação de processos (LEE \& AHN, 2008). As ações de melhoria de processos, segundo Valle \& Oliveira (2009), incorporam metodologias para otimização de processos, como metodologias da gestão da qualidade e práticas de benchmarking (ARMISTEAD \& MACHIN, 1997).

A prática de medição, monitoramento e controle de processos (P5) compreende as atividades de registrar o desempenho dos processos ao longo do tempo, suportando assim as práticas de planejamento de BPM, modelagem e melhoria de processos (HERNAUS et al., 2012; VALLE \& OLIVERA, 2009). De acordo com a ABPMP 
(ASSOCIATION..., 2009), essa prática permite verificar a efetividade das mudanças e avaliar se os objetivos estabelecidos no planejamento de BPM estão sendo atingidos.

Os modelos de ciclo de vida de BPM consistem na aplicação combinada das demais práticas discutidas anteriormente. Existe uma grande diversidade de modelos de ciclo de vida de BPM na literatura que podem ser classificados quanto ao foco de aplicação: modelos com foco na gestão de sistemas de informação e modelos com foco na gestão organizacional (VALLE \& OLIVEIRA, 2009).

\subsection{Análise de citações}

As publicações de BPM também foram analisadas quanto ao número de citações. Essa análise contemplou 29 publicações das duas bases de dados (11 publicações duplicadas nas duas bases de dados). A Tabela 2 mostra a lista de artigos mais citados na base de dados ISI WoS, enquanto a Tabela 3 traz a lista de artigos mais citados na base Scopus. A Figura 5 mostra a evolução do número de citações dos 20 artigos mais citados em cada base de dados, nela é possível notar que o número de citações começou a crescer consideravelmente no início da década de 2000.
Foi possível verificar que a grande maioria das publicações foi classificada na categoria A1. Assim foram analisadas também as publicações da categoria A2 e A3 com maior índice de citação.

Dentre as publicações com maior ICc, os temas mais abordados foram: sistemas de BPM e de gestão de workflow, arquitetura de software orientada a serviços (SOA) e modelagem de processos com foco na implementação de sistemas de informação. Embora os estudos mais citados sejam da categoria A1, foram identificados estudos das categorias A2 e A3, descritos a seguir:

- Lindsay et al. (2003) (21 $1^{\circ}$ no ranking de citações ISI WoS) apresentaram um estudo no qual discutem as definições de processos de negócio, especialmente as definições baseadas em tecnologias. Para os autores, essas definições não apresentam a real natureza dos processos de negócio, visto que as definições devem focar em como as atividades são realizadas. Os autores ainda exploram as técnicas de modelagem de processos de negócio para destacar a diferença entre o que é modelado (estático) e o que é executado (dinâmico);

Tabela 2. Lista de artigos mais citados na base de dados ISI WoS (fator de impacto JCR referente ao ano de 2011).

\begin{tabular}{|c|c|c|c|c|c|}
\hline Artigo & Periódico & IC & IC \% & JCR & ICc \\
\hline Faratin et al. (1998) & Robotics and Autonomous Systems & 330 & $9,24 \%$ & 1,056 & 678,5 \\
\hline Wooldridge et al. (2000) & Autonomous Agents and Multi-agent Systems & 289 & $8,09 \%$ & 1,213 & 639,6 \\
\hline Al-Mashari et al. (2003) & European Journal of Operational Research & 128 & $3,58 \%$ & 1,815 & 360,3 \\
\hline Grigori et al. (2004) & Computers in Industry & 111 & $3,11 \%$ & 1,529 & 280,7 \\
\hline Van der Aalst et al. (2005a) & Data \& Knowledge Engineering & 114 & $3,19 \%$ & 1,422 & 276,1 \\
\hline Van der Aalst et al. (2007) & Information Systems & 112 & $3,14 \%$ & 1,198 & 246,2 \\
\hline Jennings et al. (1996) & $\begin{array}{l}\text { International Journal of Cooperative } \\
\text { Information Systems }\end{array}$ & 79 & $2,21 \%$ & 1,278 & 180,0 \\
\hline Mendling et al. (2008) & Data \& Knowledge Engineering & 72 & $2,02 \%$ & 1,422 & 174,4 \\
\hline $\mathrm{Xu}(2011)$ & IEEE Transactions on Industrial Informatics & 40 & $1,12 \%$ & 2,99 & 159,6 \\
\hline Wang, Wang e Xu (2005) & Knowledge-based Systems & 34 & $0,95 \%$ & 2,422 & 116,3 \\
\hline Jung (2009) & Expert Systems with Applications & 29 & $0,81 \%$ & 2,203 & 92,9 \\
\hline Eshuis \& Grefen (2008) & Data \& Knowledge Engineering & 37 & $1,04 \%$ & 1,422 & 89,6 \\
\hline Wang \& Wang (2006) & Information \& Management & 27 & $0,76 \%$ & 2,214 & 86,8 \\
\hline Bae et al. (2004) & $\begin{array}{l}\text { IEEE Transactions on Knowledge and Data } \\
\text { Engineering }\end{array}$ & 32 & $0,90 \%$ & 1,657 & 85,0 \\
\hline Recker et al. (2009) & $\begin{array}{l}\text { Journal of the Association for Information } \\
\text { Systems }\end{array}$ & 31 & $0,87 \%$ & 1,667 & 82,7 \\
\hline Mutschler et al. (2008) & $\begin{array}{l}\text { IEEE Transactions on Systems Man and } \\
\text { Cybernetics Part C-Applications and Reviews }\end{array}$ & 27 & $0,76 \%$ & 2,009 & 81,2 \\
\hline Gou et al. (2003) & Computers in Industry & 28 & $0,78 \%$ & 1,529 & 70,8 \\
\hline Chen et al. (2007) & Decision Support Systems & 26 & $0,73 \%$ & 1,687 & 69,9 \\
\hline Tan et al. (2008) & $\begin{array}{l}\text { IEEE Transactions on Systems Man and } \\
\text { Cybernetics Part C-Applications and Reviews }\end{array}$ & 23 & $0,64 \%$ & 2,009 & 69,2 \\
\hline Dang et al. (2008) & Journal of Biomedical Informatics & 23 & $0,64 \%$ & 1,792 & 64,2 \\
\hline
\end{tabular}


Tabela 3. Lista de artigos mais citados na base de dados Scopus (fator de impacto SJR2 referente ao ano de 2011).

\begin{tabular}{llllll}
\hline \multicolumn{1}{c}{ Artigo } & \multicolumn{1}{c}{ Periódico } & IC & IC \% & SJR2 & ICc \\
\hline Wooldridge et al. (2000) & Autonomous Agents and Multi-Agent Systems & 330 & $9,24 \%$ & 1,056 & 678,5 \\
Cardoso et al. (2004) & Web Semantics & 289 & $8,09 \%$ & 1,213 & 639,6 \\
Al-Mashari et al. (2003) & European Journal of Operational Research & 128 & $3,58 \%$ & 1,815 & 360,3 \\
Faratin et al. (1998) & Robotics and Autonomous Systems & 111 & $3,11 \%$ & 1,529 & 280,7 \\
Van der Aalst et al. (2005a) & Data and Knowledge Engineering & 114 & $3,19 \%$ & 1,422 & 276,1 \\
Van der Aalst et al. (2007) & Information Systems & 112 & $3,14 \%$ & 1,198 & 246,2 \\
Grigori et al. (2004) & Computers in Industry & 79 & $2,21 \%$ & 1,278 & 180,0 \\
Schulz \& Oklowska (2004) & Data and Knowledge Engineering & 72 & $2,02 \%$ & 1,422 & 174,4 \\
Sun et al. (2006) & Information Systems Research & 40 & $1,12 \%$ & 2,99 & 159,6 \\
Bae et al. (2004) & IEEE Transactions on Knowledge and Data & 34 & $0,95 \%$ & 2,422 & 116,3 \\
& Engineering & 29 & $0,81 \%$ & 2,203 & 92,9 \\
Jennings et al. (1996) & International Journal of Cooperative & & & & \\
& Information Systems & 37 & $1,04 \%$ & 1,422 & 89,6 \\
Leymann et al. (2002) & IBM Systems Journal & 27 & $0,76 \%$ & 2,214 & 86,8 \\
Melão \& Pidd (2000) & Information Systems Journal & 32 & $0,90 \%$ & 1,657 & 85,0 \\
Jennings et al. (2000) & Applied Artificial Intelligence & 31 & $0,87 \%$ & 1,667 & 82,7 \\
Lohmann et al. (2008) & Data \& Knowledge Engineering & 27 & $0,76 \%$ & 2,009 & 81,2 \\
Eshuis \& Grefen (2008) & Data \& Knowledge Engineering & 28 & $0,78 \%$ & 1,529 & 70,8 \\
Recker et al. (2009) & Journal of the Association of Information & & & & \\
Dijkman et al. (2011) & Systems & 26 & $0,73 \%$ & 1,687 & 69,9 \\
Van der Aalst et al. (2005b) & Information Systems & 23 & $0,64 \%$ & 2,009 & 69,2 \\
& Computer Supported Cooperative Work & & & & \\
Mendling et al. (2008) & CSCW: an International Journal & 23 & $0,64 \%$ & 1,792 & 64,2 \\
\hline Data \& Knowledge Engineering & & &
\end{tabular}

Nota: Artigos classificados em ordem decrescente de índice corrigido de citações.
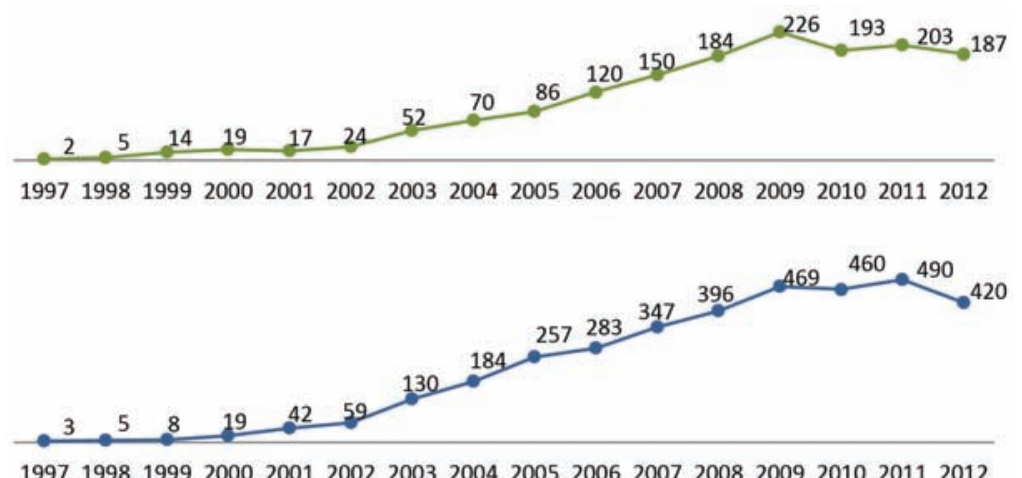

Figura 5. Citações recebidas pelos artigos mais citados nas bases ISI WoS e Scopus, respectivamente.

- Elzinga et al. (1995) (23ํ no ranking de citações Scopus e $24^{\circ}$ no ISI WoS) mencionaram BPM como uma abordagem para a melhoria contínua de processos de negócio como, por exemplo os processos de manufatura e marketing. Os autores ainda apresentam uma metodologia para melhoria contínua de processos baseada em práticas de benchmarking, planejamento, seleção, descrição, melhoria de processos e implementação;
- Vergidis et al. (2008) (43 no ranking de citações ISI WoS) conduziram uma revisão da literatura seguida de um levantamento do tipo survey em empresas prestadoras de serviço para analisar a diferença entre a teoria e prática de BPM. Foram avaliados aspectos como: percepção e compreensão sobre os processos de negócio; estrutura organizacional; modelagem e extração de dados de processos; análise, melhoria e conformidade de processos. 
Os resultados mostraram que as organizações possuem uma compreensão sobre o que é e quais são os benefícios de BPM, entretanto elas não possuem conhecimentos e recursos necessários para a modelagem e melhoria de processos em uma estrutura orientada a processos. Além disso, as ações de modelagem e melhoria ocorrem de forma isolada e por meio de técnicas manuais, o que não permite que BPM atinja todo seu potencial quanto à melhoria dos processos;

- Recker (2010) (48 no ranking de citações Scopus) investigou a aceitação da notação BPMN para a modelagem de processos de negócio, destacando suas limitações e oportunidades de melhoria quanto à metodologia de modelagem;

- Trkman (2010) (49 no ranking de citações Scopus) analisou os fatores de sucesso de BPM, com destaque para o treinamento e capacitação de recursos e maior autonomia para os funcionários em relação à execução e autorização dos processos;

- Armistead et al. (1999) (28 no ranking de citações Scopus) analisaram BPM como um modelo de gestão que deve atuar tanto nos níveis estratégicos como nos níveis operacionais. Os autores destacam sete temas que devem ser considerados em BPM: escolha e direcionamento estratégico; desenho organizacional; maximização da cadeia de valor do mercado; gestão de desempenho; coordenação organizacional; gestão de conhecimento; e cultura organizacional;

- Hung (2006) (44 no ranking de citações Scopus) analisou dois conceitos-chave de BPM e os seus efeitos no desempenho organizacional por meio de um estudo empírico. Segundo o autor, os conceitos-chave, que são alinhamento dos processos e envolvimento de pessoas, estão diretamente relacionados com o desempenho positivo das organizações.

Assim, como a análise de trabalhos mais citados permitiu identificar os trabalhos representativos da área, a análise de redes de artigos para referência permitiu identificar estudos relevantes e a existência de um pequeno agrupamento bibliométrico, composto por Smart et al. (2009), Maddern et al. (2007), Llewellyn \& Armistead (2000), Armistead et al. (1999), Kumar et al. (2008), Hung (2006), Elzinga et al. (1995), Trkman (2010), Vergidis et al. (2008), Lindsay et al. (2003), Melão \& Pidd (2000) e Mutschler et al. (2008), conforme mostra a Figura 6. O conteúdo da maioria desses trabalhos já foi analisado durante a análise das citações, visto

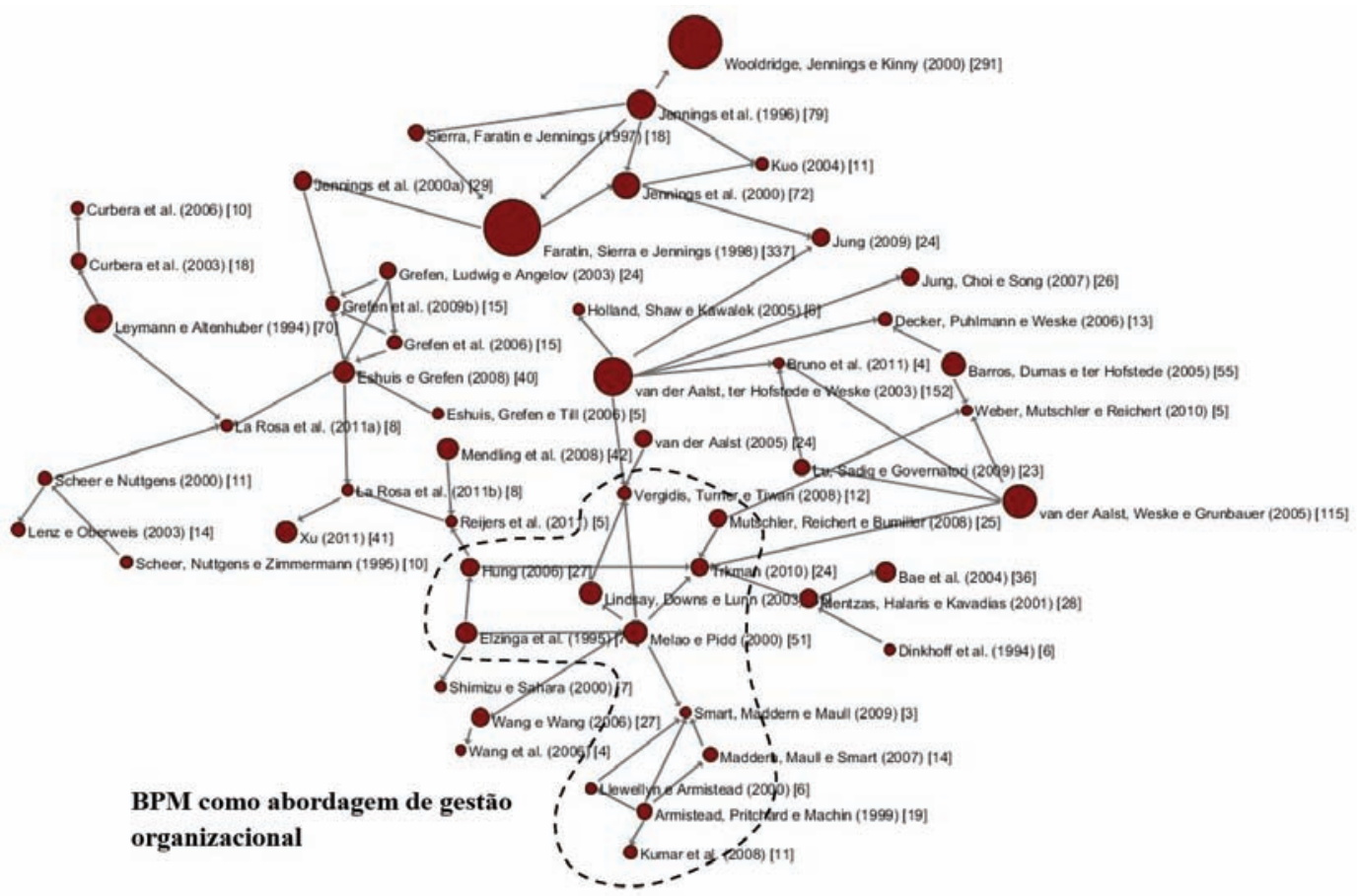

Figura 6. Rede de artigos para referência da base ISI WoS. Nota 1: Critério de corte de três citações por artigo. Nota 2: A variação de tamanho representa as citações do artigo (maior tamanho = maior índice de citações), os números entre colchetes correspondem ao número de citações do artigo e as setas indicam o fluxo de informação, ou seja, a origem da seta é a referência e o destino é o artigo. 
que esses trabalhos apresentam um elevado número de citações.

Smart et al. (2009) propuseram um modelo para a compreensão de BPM. Segundo os autores, a BPM possui elementos que podem ser classificados em elementos de aplicação e elementos teóricos. Os elementos de aplicação são: estratégia de processos, arquitetura de processos, medição de processos, responsabilidade de processos e melhoria de processos. Os elementos teóricos são: gestão de macro processos, gestão consciente de processos e centralidade por processo. Cabe destacar que esse modelo não é um modelo de ciclo de vida de BPM mas um modelo teórico, que auxilia na compreensão dos principais elementos de BPM.

Maddern et al. (2007) avaliaram os drivers para a satisfação do cliente, analisando o impacto de BPM na qualidade de serviços e na satisfação dos clientes. Kumar et al. (2008) estudaram a relação entre BPM e satisfação do cliente e seu artigo compreende uma extensão do trabalho de Maddern et al. (2007).

Llewellyn \& Armistead (2000), por sua vez, analisaram a existência de capital social em processos de negócio específicos, de modo a auxiliar na gestão dos processos com a manutenção do capital social, que pode ser entendido como a soma de recursos ao longo dos processos, que são derivados das redes de colaboração.

Nota-se que esse grupo não está isolado, já que alguns autores se relacionam com trabalhos que têm como foco sistemas de informação e gestão de workflow, como é o caso dos trabalhos de Hung (2006), Vergidis et al. (2008), Melão \& Pidd (2000) e Mutschler et al. (2008). Ao analisar os estudos, foi possível identificar que os assuntos compartilhados entre estudos das categorias A1 e os estudos do grupo são modelagem de processos (VERGIDIS et al., 2008; MELÃO \& PIDD, 2000) e a relação entre a teoria de BPM e a aplicação em sistemas de informação (HUNG, 2006; MELÃO \& PIDD, 2000; MUTSCHLER et al., 2008).

Outra rede analisada foi a de cocitação. A rede de cocitação apresenta trabalhos que foram citados conjuntamente, o que permite analisar o grau de similaridade entre as referências dos artigos da amostra, bem como a formação de clusters por tópico de estudo. Essa análise permite compreender o quadro teórico de uma área e mapear as afinidades entre grupos de pesquisadores e como eles se relacionam. Foram elaboradas duas redes de cocitação, uma para a amostra extraída da base ISI WoS e outra para a amostra extraída da base Scopus. Em seguida foram calculados três indicadores para auxiliar na análise das redes: densidade da rede, índice de centralidade e índice de intermediação.

A densidade da rede elaborada na base de dados ISI WoS foi de $4 \%$ (número de nós = 100; número de relações $=200$ ), o que mostra o baixo número de relações entre os artigos da rede. No que diz respeito à intermediação, foi possível identificar dois trabalhos importantes: Smith \& Fingar (2003) descrevem em seu livro as ondas de BPM, que compreendem a análise de métodos e procedimentos $\left(1^{a}\right.$ onda), a reengenharia e uso de sistemas ERP $\left(2^{a}\right.$ onda) e a extensão das tecnologias e técnicas de gestão de BPM ( $3^{\text {a }}$ onda); e Dumas et al. (2005), que estudaram a interface entre sistemas de informação de BPM e pessoas. Esses dois trabalhos apresentam uma importante função de conexão entre o grupo de trabalhos sobre BPR e TQM e as demais publicações da rede.

A densidade dessa rede foi de $12 \%$ (n. de nós $=130 ;$ n. de relações $=987$ ), ela pode ser considerada uma rede pouco densa. Isso confirma a existência de grupos de atores que não compartilham diretamente informações, confirmando o caráter multidisciplinar de BPM (KO et al, 2009). No que diz respeito ao índice de intermediação, as publicações que se destacaram foram: Sun et al. (2006), Zairi (1997), Davenport (1993), van der Aalst (1998), Reichert \& Dadam (1998) e Al-Mashari \& Zairi (2000).

A Figura 7 mostra a rede de cocitação elaborada a partir dos metadados extraídos da base ISI WoS. Foi possível identificar dois clusters: um tipicamente composto por trabalhos com foco em BPR e TQM (C1); outro composto por trabalhos com foco em sistemas de informação e tecnologia de BPM (C2). Na Figura 8, que mostra a rede de cocitação elaborada a partir dos metadados extraídos da base Scopus, foi possível identificar, além desses dois clusters (C3 e C4), mais dois clusters com menor densidade que tratam do tema BPM.

Foram identificadas similaridades entre as duas redes de cocitação. No que diz respeito aos trabalhos duplicados e aos grupos destacados (destacados nas Figuras 7 e 8), alguns estudos relacionam-se às origens de BPM, compreendendo os movimentos da reengenharia (BPR - Business Process Reengineering) e da Gestão da Qualidade Total (TQM - Total Quality Management) (GARVIN, 1995).

O movimento da BPR foi introduzido por Hammer (1990) e Hammer \& Champy (1993) e sua principal característica é a mudança radical nos processos de negócio. Segundo os autores, é a reengenharia que determina o que são suas atividades e como a organização deve realiza-las. Outros trabalhos, como os de Davenport \& Short (1990) e Davenport (1993) também estão relacionados ao tema de BPR.

O movimento TQM, ao contrário da reengenharia, prega a mudança incremental e contínua nos processos de negócio (ZAIRI \& SINCLAIR, 1995). Harrington (1998) e Harmon (2007) são o referencial 
teórico desse movimento, cujo foco é a melhoria incremental e contínua de processos.

Elzinga et al. (1995) desenvolveram um modelo para a aplicação de BPM em indústrias. Os autores consideraram também as abordagens de BPR e TQM em uma estrutura típica de ciclo de vida de processos de negócio. As etapas desse modelo incluem: Benchmarking, Preparação, Seleção de processos, Descrição de processos, Quantificação de processos, Seleção de melhoria de processo e Implementação.

Zairi (1997) e Lee \& Dale (1998) apresentam conceitos e as características de BPM. Segundo os

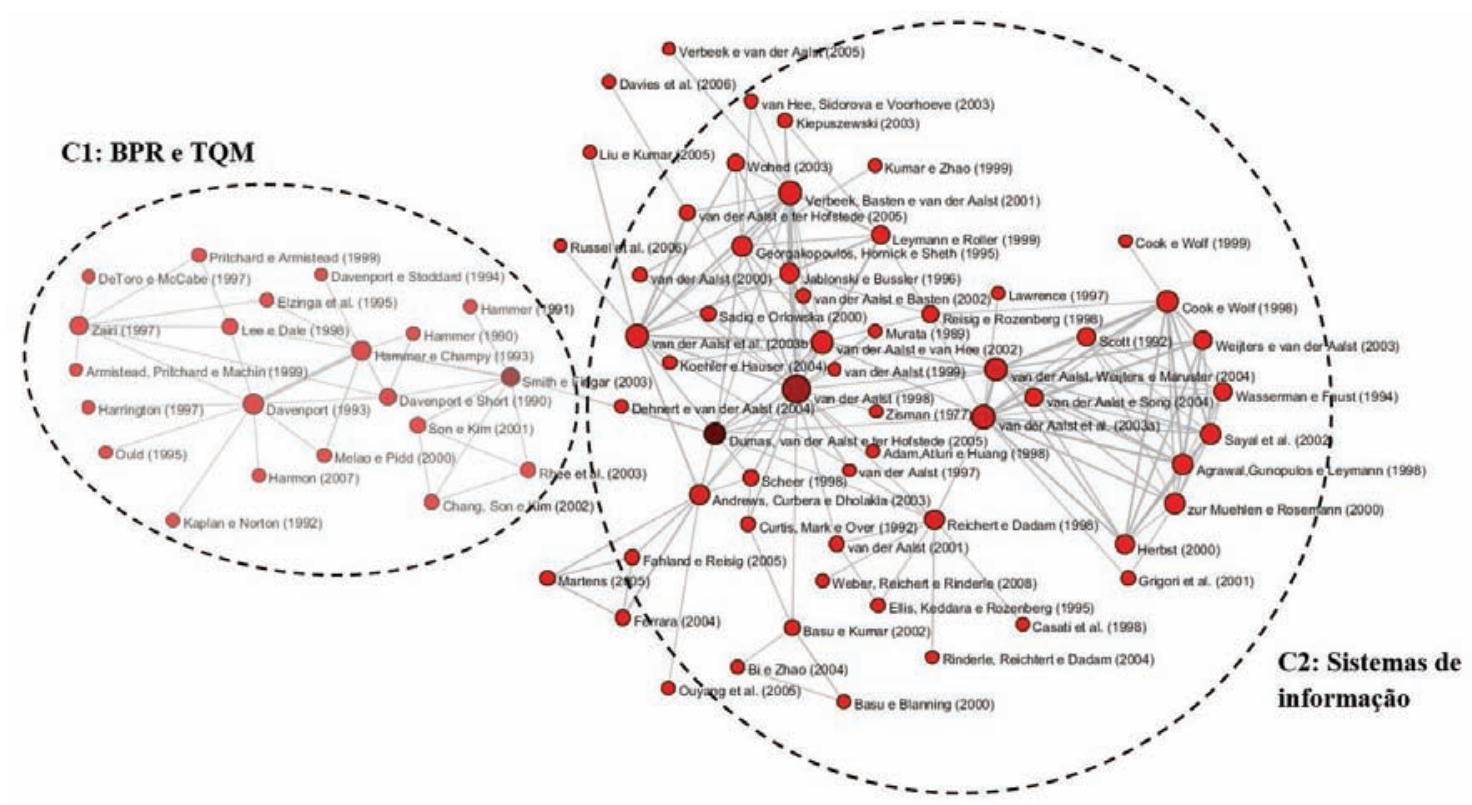

Figura 7. Rede de cocitação para a base ISI WoS; cluster de artigos sobre BPR e TQM (C1); e cluster de artigos com foco em sistemas de informação (C2). Nota 1: Critério de corte de três citações. Nota 2: A variação de tamanho representa o índice de centralidade (maior tamanho = maior índice de centralidade), a variação de cor representa o índice de intermediação (mais escuro = maior índice de intermediação) e a espessura das ligações representa a força dos relacionamentos (maior espessura $=$ maior o número de relações compartilhadas).

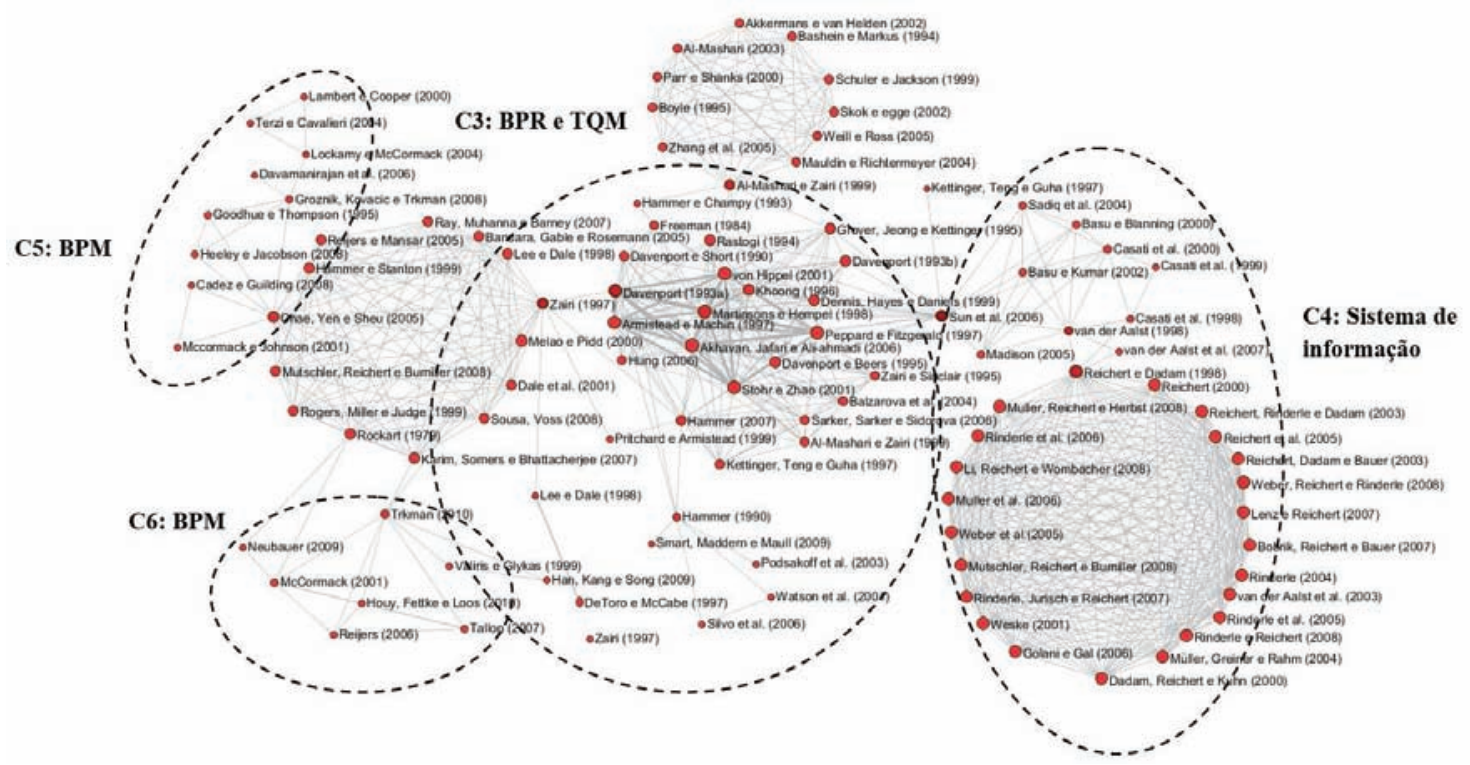

Figura 8. Rede de cocitação para a base Scopus; cluster de artigos com dobre BPR e TQM (C3); cluster de artigos com foco em sistemas de informação (C4); e clusters de artigos sobre BPM (C5, C6). Nota 1: Sem critério de corte. Nota 2: A variação de tamanho representa o índice de centralidade (maior tamanho = maior índice de centralidade), a variação de cor representa o índice de intermediação (mais escuro = maior índice de intermediação) e a espessura das ligações representa a força dos relacionamentos (maior espessura = maior o número de relações compartilhadas). 
autores, BPM inclui: mapeamento e documentação de atividades; orientação ao cliente; dependência de sistemas e procedimentos documentados para assegurar a coerência; medição de desempenho; foco na melhoria contínua; abordagem para a mudança de cultura.

Armistead et al. (1999) apresentam a relação entre BPM e efetividade organizacional, destacando a importância de alinhamento estratégico com esse tipo de abordagem. Segundo os autores, alinhar os processos de negócio em uma estrutura gerencial de BPM orientada pelo planejamento estratégico permite que as organizações atinjam seus objetivos.

Pritchard \& Armistead (1999) avaliaram, por meio de survey e estudo de caso, a aplicação de BPM em organizações e identificaram fatores relevantes para a implementação bem-sucedida de BPM. Dentre esses fatores, destacam-se: alinhamento estratégico de BPM, clareza quanto à abordagem de BPM, integração entre os níveis operacionais, táticos e estratégicos da organização, treinamento de pessoal e gestão do conhecimento.

A partir desses resultados é possível estabelecer algumas afirmações:

- BPM tem suas origens principalmente nas abordagens de BPR, TQM e sistemas de informação, nas quais as duas primeiras, embora sejam abordagens com características distintas e opostas, são citadas pelos mesmos artigos com certa frequência (resultados encontrados em duas bases de dados renomadas e com critério de corte elevado para a base ISI WoS);

- BPM é uma abordagem com origem multidisciplinar. Pode-se afirmar que existem ao menos duas "escolas", uma com foco na gestão organizacional, que tem como origens BPR e TQM, e outra com foco nas tecnologias de BPM, que têm como origem a área de sistemas de informação e computação. Entretanto, essas "escolas" tendem a compartilhar pouco conhecimento, já que as redes apresentaram baixa densidade e existem poucos trabalhos com alto índice de intermediação (capacidade de conectar grupos distintos). Uma maior contribuição entre essas "escolas" certamente resultaria em avanços maiores para a área de BPM;

- BPM está se consolidando como uma área de pesquisa, pois a formação de pequenos clusters de artigos tipicamente sobre BPM na rede de cocitação (C5 e C6) indica que cada vez mais os artigos de BPM são citados em conjunto.

\section{Conclusões}

A análise de publicações permitiu identificar os periódicos mais relevantes para o tema BPM, como Business Process Managament Journal, Knowlegde and Process Management e Total Quality Management \& Business Excellence. Esse resultado direciona os principais meios de publicação científica de BPM, já que essa abordagem demonstrou ser multidisciplinar.

As publicações de BPM com foco na abordagem de gestão do ciclo de vida de processos e de gestão organizacional, a partir de 2006, representam em média $20 \%$ do volume de publicações anual de artigos relacionados à BPM. Apesar do crescimento do volume de produção, acredita-se existir a possibilidade de aumentar esse volume, visto que as publicações não esgotam o tema BPM.

No que diz respeito às práticas de BPM, a modelagem de processos de negócio e a implementação de processos e sistemas de apoio são as mais abordadas nos estudos da amostra. Isso pode ser reflexo das publicações com foco em sistemas de informação, que normalmente fazem uso da modelagem de processos para a implementação de sistemas de BPM (software) e arquitetura orientada a serviços. Entretanto, uma atividade pouco abordada nos estudos foi planejamento de BPM. Essa prática é responsável por fazer a ligação entre os processos de negócio e o planejamento estratégico da organização. Isso indica que BPM, devido suas origens em BPR e TQM, ainda é aplicada como uma abordagem bottom-up, com melhorias incrementais nos níveis operacionais. Nesse sentido, alguns autores como Trkman (2010) e Smart et al. (2009) apresentam trabalhos que indicam a importância de BPM ser aplicada como uma abordagem top-down.

Os resultados das redes de bibliometria confirmam a multidisciplinaridade de BPM, bem como a sua relação com a gestão da qualidade, a reengenharia e a área de sistemas de informação, mas indicam também que essas áreas científicas tendem a evoluir de forma isolada. Além disso, mostram a existência de dois grandes grupos: um relacionado à aplicação de BPM na modelagem de processos e implementação de sistemas de informação, outro relacionado à gestão da organização como um todo. Esse segundo grupo tem como origens os movimentos de BPR e TQM. Assim, uma integração entre essas áreas poderiam produzir grandes avanços para a prática de BPM.

As análises utilizadas nesta pesquisa permitiram a estruturação da literatura de BPM. Esse resultado pode orientar pesquisadores e gestores interessados na área, bem como permitir a aproximação entre as duas "escolas" de BPM. 


\section{Agradecimentos}

Agradecemos à CAPES e ao CNPq, pelo suporte ao projeto de pesquisa, e aos revisores pelos comentários que permitiram a evolução e melhoria desta pesquisa.

\section{Referências}

AL-MASHARI, M.; ZAIRI, M. Revisiting BPR: a holistic review of practice and development. Business Process Management Journal, v. 6, n. 1, p. 10-42, 2000. http:// dx.doi.org/10.1108/14637150010283045

AL-MASHARI, M.; AL-MUDIMIGH A.; ZAIRI, M. Enterprise resource planning: a taxonomy of critical factors. European Journal of Operational Research, v. 146, n. 2, p. 352-364, 2003. http://dx.doi. org/10.1016/S0377-2217(02)00554-4

ARMISTEAD, C.; PRITCHARD, J. P.; MACHIN, S. Strategic business process management for organisational effectiveness. Long Range Planning, v. 32, n. 1, p. 96-106, 1999. http://dx.doi.org/10.1016/ S0024-6301(98)00130-7

ARMISTEAD, C.; MACHIN, S. Implications of business process management for operations management, International Journal of Operations \& Production Management, v. 17, n. 9, p. 886-898, 1997. http:// dx.doi.org/10.1108/01443579710171217

ASSOCIATION OF BUSSINESS PROCESS MANAGEMENT PROFESSIONALS - ABPMP. Guia para o Gerenciamento de Processos de Negócio: Corpo Comum de Conhecimento. [S.1], 2009.

BAE, J. et al. Automatic control of workflow processes using ECA rules. IEEE Transactions on Knowledge and Data Engineering, v. 16, n. 8, p. 1010-1023, 2004. http://dx.doi.org/10.1109/TKDE.2004.20

BIOLCHINNI, J. et al. Systematic review in software engineering. Rio de Janeiro, 2005. (Report).

BOARDMAN, J. Corporate intranets and business process management: a challenge for systems engineering. Computing \& Control Engineering Journal, v. 8, n. 6, p. 245-256, 1997. http://dx.doi.org/10.1049/ cce: 19970604

BORNER, K.; SANYAL, S.; VESPIGNAN, A. Network science. In: CRONIN, B. (Ed.). Annual Review of Information Science \& Technology. Medford: Information Today; American Society for Information Science and Technology, 2007. p. 537-607. v. 41. cap. 12.

CARDOSO, J. et al. Quality of service for workflows and web service processes. Web Semantics, v. 1, n. 3, p. 281-308, 2004. http://dx.doi.org/10.1016/j. websem.2004.03.001

CARVALHO, M. M.; FLEURY, A.; LOPES, A. P. An overview of the literature on technology roadmapping (TRM): contributions and trends. Technological Forecasting \& Social Change, v. 80, n. 7, p. 1418-1437, 2013. http://dx.doi.org/10.1016/j.techfore.2012.11.008 CHEN, M.; ZHANG, D.; ZHOU, L. Empowering collaborative commerce with Web services enabled business process management systems. Decision Support Systems, v. 43, n. 2, p. 530-546, 2007.

CULNAN, M. J.; O'REILLY, C. A.; CHATMAN, J.A. Intellectual structure of research in organizational behavior, 1972- 1984: a cotitation analysis. Journal of the American Society for Information Science and Technology, v. 41, n. 6, p. 453-458, 1990. http://dx.doi.org/10.1002/(SICI)10974571(199009)41:6<453::AID-ASI13>3.0.CO;2-E

DANG, J. et al. Ontological knowledge framework for adaptive medical workflow. Journal of Biomedical Informatics, v. 41, n. 5, p. 829-836, 2008. PMid:18602872. http://dx.doi.org/10.1016/j. jbi.2008.05.012

DAVENPORT, T. H. Process innovation: reeginering work though information technology. Harvard Business Press, 1993.

DAVENPORT, T. H. The coming commoditization of process. Harvard Business Review, v. 83, n. 6, p. 100-108, 2005. PMid:15942994

DAVENPORT, T. H.; SHORT, J. E. The new industrial engineering: information technology and business process redesign. Sloane Management Review, v. 31, n. 4, p. 11-27, 1990.

DEBRUIN, T.; DOEBELI, G. BPM as an organisational approach: the experience of an Australian transport provider. In: ROSEMANN, M.; VON BROCKE, J. (Ed.). Handbook on Business Process Management. Berlin: Springer, 2009. v. 1

DIJKMAN, R. et al. Similarity of business process models: metrics and evaluation. Information Systems, v. 36, n. 2, p. 498-516, 2011.

DUMAS, M.; VAN DER AALST, W. M.; TER HOFSTEDE, A. H. Process aware information systems: bridging people and software through process technology. Wiley-Interscience, 2005.

ELZINGA, D. J. et al. Business process management: survey and methodology. IEEE Transactions on Engineering Management, v. 42, n. 2, p. 119-128, 1995.

ESHUIS, R.; GREFEN, P. Constructing customized process views. Data \& Knowledge Engineering, v. 64, n. 2, p. 419-438, 2008. http://dx.doi.org/10.1016/j. datak.2007.07.003

FARATIN, P.; SIERRA, C.; JENNINGS, N. R. Negotiation decision functions for autonomous agents. Robotics and Autonomous Systems, v. 24, n. 3-4, p. 159-182, 1998. http://dx.doi.org/10.1016/S0921-8890(98)00029-3

GARFIELD, E. From bibliographic coupling to co-citation analysis via algorithmic historiobibliography: a citationist's tribute to Belver C. Griffith. [S.1.]., 2001.

GARVIN, D. A. Leveraging processes for strategic advantage. Harvard Business Review, v. 73, n. 5, p. 77-90, 1995.

GIPP, B.; BEEL, J. Citation Proximity Analysis (CPA): a new approach for identifying related work based on Co-Citation Analysis. In: LARSEN, B.; LETA, J. (Ed.). INTERNATIONAL CONFERENCE ON SCIENTOMETRICS AND INFORMETRICS 
(ISSI'09), 12., 2009, Rio de Janeiro. Proceedings... p. 571-575. v. 2.

GRIGORI, D. et al. Business process intelligence. Computers in Industry, v. 53, n. 3, p. 321-343, 2004.

HAMMER, M. Reengineering work: don't automate, obliterate. [S.1.]., 1990.

HAMMER, M.; CHAMPY, J. Reengineering in the corporation: a manifesto for business revolution. Harper Business, 1993.

HAN, K. H.; KANG, J. G.; SONG, M. Two-stage process analysis using process-based performance measurement framework and business process simulation. Expert Systems with Applications, v. 36, n. 3, p. 7090-7086, 2009. http://dx.doi.org/10.1016/j.eswa.2008.08.035

HARMON, P. Business process change: a guide for business managers and BPM and Six Sigma professionals. 2nd ed. San Francisco: Morghan Kaufman, 2007.

HARMON, P. The scope and evolution of Business Process Management, In: VOM BROCKE, J.; ROSEMANN, M. (Ed.). Handbook on Business Process Management. Heidelberg: Springer, 2010. v. 1. Introduction, methods, and information systems.

HARRINGTON, H. J. Performance improvement: the rise and fall of reeginneering. The TQM Magazine, v. 10, n. 2, p. 69-71, 1998.

HERNAUS, T.; BACH, M.; VUKSIC, V. Influence of strategic approach to BPM on financial and non-financial performance. Baltic Journal of Management, v. 7, n. 4, p. 376-396, 2012.

HUNG, R. Y. Y. Business Process Management as competitive advantage: a review and empirical study, Total Quality Management and Business Excellence, v. 17, n. 1, p. 21-40, 2006.

JENNINGS, N. R. et al. Agent-based business process management. International Journal of Cooperative Information Systems, v. 5, n. 2-3, p. 105-130, 1996. http://dx.doi.org/10.1142/S0218843096000051

JENNINGS, N. R. et al. Autonomous agents for business process management. Applied Artificial Intelligence, v. 14, n. 2, p. 145-189, 2000.

JUNG, J. J. Semantic business process integration based on ontology alignment. Expert Systems with Applications, v. 36, n. 8, p. 11013-11020, 2009.

KO, R. K. L.; LEE, S. S. G.; LEE, E. W. Business process management (BPM) standards: a survey. Business Process Management Journal, v. 15, n. 5, p. 744-791, 2009.

KOHLBACHER, M. The effects of process orientation: a literature review. Business Process Management Journal, v. 16, n. 1, p. 135-152, 2010.

KUMAR, V. et al. Alternative perspectives on service quality and customer satisfaction: the role of BPM. International Journal of Service Industry Management, v. 19, n. 2, p. 176-187, 2008.

LEE, R. G.; DALE, B. G. Business process management: a review and evaluation. Business Process Management Journal, v. 4, n. 3, p. 214-225, 1998. http://dx.doi. org/10.1108/14637159810224322
LEE, S.; AHN, H. Assessment of process improvement from organizational change. Information \& Management, v. 45, n. 5, p. 270-280 2008.

LEYMANN, F.; ROLLER, D.; SCHMIDT, M. T. Web services and business process management. IBM Systems Journal, v. 41, n. 2, p. 198-211, 2002.

LINDSAY, A.; DOWNS, D.; LUNN, K. Business processes: attempts to find a definition. Information and Software Technology, v. 45, n. 15, p. 1015-1019, 2003. http://dx.doi.org/10.1016/S0950-5849(03)00129-0

LLEWELLYN, N.; ARMISTEAD, C. Business process management management. International Journal, v. 11 , n. 3, p. 225-243, 2000.

LOHMANN, N. et al. Analyzing interacting WS-BPEL processes using flexible model generation. Data \& Knowledge Engineering, v. 64, n. 1, p. 38-54, 2008.

LOPES, A. P. V. B. V.; CARVALHO, M. M. Evolução da literatura de inovação em relações de cooperação: um estudo bibliométrico num período de vinte anos. Gestão e Produção, v. 19, n. 1, p. 203-217, 2012. http://dx.doi.org/10.1590/S0104-530X2012000100014

MADDERN, H.; MAULL, R.; SMART, P. A. Customer satisfaction and service quality in UK financial services. International Journal of Operations \& Production Management, v. 27, n. 9, p. 999-1019, 2007.

MELÃO, N.; PIDD, M. A conceptual framework for understanding business processes and business process modelling. Information Systems Journal, v. 10, n. 2 , p. $105-129,2000$. http://dx.doi. org/10.1046/j.1365-2575.2000.00075.x

MENDLING, J. et al. Detection and prediction of errors in EPCs of the SAP reference model. Data and Knowledge Engineering, v. 64, n. 1, p. 312-329, 2008.

MUTSCHLER, B.; REICHERT, M.; BUMILLER, J. Unleashing the effectiveness of process-oriented information systems: problem analysis, critical success factors, and implications. IEEE Transactions on Systems, Man, and Cybernetics, Part C: Applications and Reviews, v. 38, n. 3, p. 280-291, 2008.

PEDRINACI, C. et al. Semantic business process management: Scaling up the management of business processes. In: 2008 IEEE INTERNATIONAL CONFERENCE ON SEMANTIC COMPUTING (ICSC'08), 2008, Santa Clara. Proceedings...

PILKINGTON, A.; LISTON-HEYES, C. Is production and operations management a discipline? A citation/ cocitation study. International Journal of Operations \& Production Management, v. 19, n. 1, p. 7-20, 1999.

PRITCHARD, J.; ARMISTEAD, C. Business process management: lessons from European business. Business Process Management Journal, v. 5, n. 1, p. 10-32, 1999.

RECKER, J. et al. Business process modeling: a comparative analysis. Journal of the Association for Information Systems, v. 10, n. 4, p. 333-363, 2009.

RECKER, J. Opportunities and constraints: the current struggle with BPMN. Business Process Management Journal, v. 16, n. 1, p. 181-201, 2010.

REICHERT, M.; DADAM, P. ADEPTflex: supporting dynamic changes of workflows without losing 
control. Journal of Intelligent Information Systems, v. 10, n. 2, p. 93-129, 1998. http://dx.doi. org/10.1023/A:1008604709862

SCHULZ, K. A.; OKLOWSKA, M. E. Facilitating cross-organisational workflows with a workflow view approach. Data and Knowledge Engineering, v. 51, n. 1, p. 109-147, 2004.

SCI2 TEAM. Science of Science (Sci2) Tool. Indiana University and SciTech Strategies, 2009. Disponível em: 〈http://sci2.cns.iu.edu>.

SKRINJAR, R.; TRKMAN, P. Increasing process orientation with business process management: critical practices. International Journal of Information Management, v. 33, p. 48-60, 2013.

SMART, P. A.; MADDERN, H.; MAULL, R. S. Understanding Business Process Management: implications for theory and practice. British Journal of Management, v. 20, p. 491-507, 2009.

SMITH, H.; FINGAR, P. Business process management: the third wave. Meghan-Kiffer Press, 2003.

SUN, S. X. et al. Formulating the data-flow perspective for business process management. Information Systems Research, v. 17, n. 4, p. 374-391, 2006.

TAN, W. et al. Business process intelligence system for enterprise process performance management. IEEE Transactions On Systems Man And Cybernetics Part C: Applications and Reviews, v. 38, n. 6, p. 745-756, 2008.

TRKMAN, P. The critical success factors of business process management. International Journal of Information Management, v. 30, n. 2, 125-134, 2010.

VALLE, R.; OLIVEIRA, S. B. Análise e Modelagem de Processos de Negócio: Foco na Notação Bpmn. São Paulo: Atlas, 2009.

VAN DER AALST, W. M. P.; WESKE, M.; GRUNBAUER, D. Case handling: a new paradigm for business process support. Data and Knowledge Engineering, v. 53, n. 2, p. 129-162, 2005a. http://dx.doi.org/10.1016/j. datak.2004.07.003

VAN DER AALST, W. M. P.; REIJERS, H. A.; SONG, M. Discovering social networks from event logs. Computer Supported Cooperative Work, v. 14, n. 6, p. 549-593, 2005b.
VAN DER AALST, W. M. P. The application of Petri Nets to workflow management. Journal Of Circuits, Systems And Computers, v. 8, n. 1, p. 21-66, 1998. http://dx.doi.org/10.1142/S0218126698000043

VAN DER AALST, W. M. P. et al. Business process mining: an industrial application. Information Systems, v. 32, n. 5, p. 713-732, 2007.

VERGIDIS, K.; TURNER, C. J.; TIWARI, A. Business process perspectives: theoretical developments vs. realworld practice. International Journal of Production Economics, v. 114, n. 1, p. 91-104, 2008. http://dx.doi. org/10.1016/j.ijpe.2007.12.009

VOLKNER, P.; WERNERS, B. A decision support system for business process planning. European Journal of Operational Research, v. 125, n. 3, p. 633-647, 2000. http://dx.doi.org/10.1016/S0377-2217(99)00273-8

WANG, M. H.; WANG, H. Q. From process logic to business logic: a cognitive approach to business process management. Information \& Management, v. 43, n. 2, p. 179-193, 2006.

WANG, M. H.; WANG, H. Q.; XU, D. M. The design of intelligent workflow monitoring with agent technology. Knowledge-Based Systems, v. 18, n. 6, p. 257-266, 2005.

WOOLDRIDGE, M.; JENNINGS, N. R.; KINNY, D. The Gaia methodology for agent-oriented analysis and design. Autonomous Agents and Multi-Agent Systems, v. 3, n. 3, p. 285-312, 2000.

XU, L. D. Enterprise systems: state-of-the-art and future trends. IEEE Transactions on Industrial Informatics, v. 7, n. 4, p. 630-640, 2011. http://dx.doi.org/10.1109/ TII.2011.2167156

ZAIRI, M. Business Process Management: a boundaryless approach to modern competitiveness. Business Process Management Journal, v. 3, n. 1, p. 64-80, 1997. http:// dx.doi.org/10.1108/14637159710161585

ZAIRI, M.; SINCLAIR, D. Business Process re-engineering and process management. Business Process Re-engineering \& Management Journal, v. 1, n. 1, p. 8-30, 1995. http://dx.doi. org/10.1108/14637159510798248 